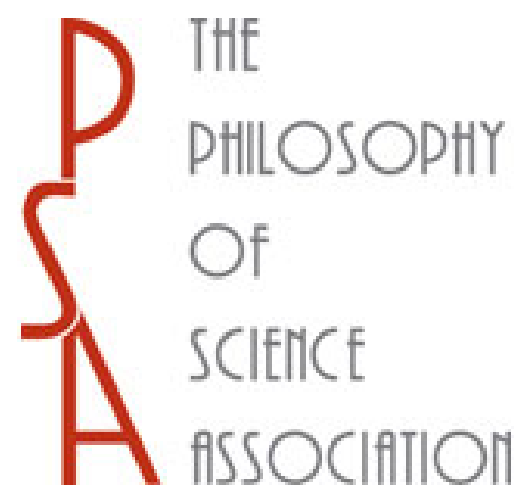

There Is No Special Problem of Mindreading in Nonhuman Animals Author(s): Marta Halina

Source: Philosophy of Science, Vol. 82, No. 3 (July 2015), pp. 473-490

Published by: The University of Chicago Press on behalf of the Philosophy of Science Association Stable URL: http://www.jstor.org/stable/10.1086/681627

Accessed: 02/11/2015 12:12

Your use of the JSTOR archive indicates your acceptance of the Terms \& Conditions of Use, available at http://www.jstor.org/page/info/about/policies/terms.jsp

JSTOR is a not-for-profit service that helps scholars, researchers, and students discover, use, and build upon a wide range of content in a trusted digital archive. We use information technology and tools to increase productivity and facilitate new forms of scholarship. For more information about JSTOR, please contact support@jstor.org. 


\title{
There Is No Special Problem of Mindreading in Nonhuman Animals
}

\author{
Marta Halina*†
}

There is currently a consensus among comparative psychologists that nonhuman animals are capable of some forms of mindreading. Several philosophers and psychologists have criticized this consensus, however, arguing that there is a "logical problem" with the experimental approach used to test for mindreading in nonhuman animals. I argue that the logical problem is no more than a version of the general skeptical problem known as the theoretician's dilemma. As such, it is not a problem that comparative psychologists must solve before providing evidence for mindreading.

1. Introduction. Adult humans use information about the cognitive states of other agents to predict and explain behavior. This ability is known as "mindreading." " Over 30 years ago, Premack and Woodruff (1978) posed

Received January 2014; revised October 2014.

*To contact the author, please write to: Department of History and Philosophy of Science, University of Cambridge, Free School Lane, Cambridge CB2 3RH, UK; e-mail: mh801 (a) cam.ac.uk.

$\dagger$ This paper was presented to the Philosophy of Science Research Group at Washington University in St. Louis, Missouri. I thank the members of that group for their helpful comments and discussion. I also thank William Bechtel, Nancy Cartwright, and C. Kenneth Waters for discussions that led to the development of the main ideas presented here, Carl Craver for detailed comments on the penultimate draft, and three anonymous reviewers for their careful and constructive feedback.

The title of this paper is inspired by Callender and Cohen's article "There Is No Special Problem about Scientific Representation" (2006). Callender and Cohen argue that the problems of scientific representation are just the problems of representation in general and thus that the proposed solutions for the latter can be applied to the former. Similarly, I argue that the problems involved in establishing the claim that nonhuman animals mindread are no different from the problems involved in establishing any theoretical claim in science. Thus, the proposed solutions to the latter can be applied to the former.

1. I follow Nichols and Stich (2003) in preferring this term over others, such as "theory of mind," as it is more theoretically neutral.

Philosophy of Science, 82 (July 2015) pp. 473-490. 0031-8248/2015/8203-0007\$10.00 Copyright 2015 by the Philosophy of Science Association. All rights reserved. 
the question, do chimpanzees mindread? Since then, comparative psychologists have conducted numerous experiments aimed at addressing this question. Recently, they have proposed an answer: chimpanzees mindread with respect to some psychological states, such as intentions and perceptions (Call and Tomasello 2008).

Over the past few years, the claim that chimpanzees mindread has been heavily criticized by a group of psychologists and philosophers led by Daniel Povinelli (Povinelli and Vonk 2003, 2004; Vonk and Povinelli 2006; Penn and Povinelli 2007, 2009, 2013; Penn, Holyoak, and Povinelli 2008; Penn 2011). These skeptics argue that the experimental approach currently used in comparative psychology to test for mindreading in nonhuman animals cannot provide evidence for this ability even in principle. The problem, they argue, is that this approach is incapable of rejecting the hypothesis that nonhuman animals solve mindreading tasks on the basis of reasoning about observable regularities alone. It is not until this alternative hypothesis is rejected that comparative psychologists are justified in claiming that they have evidence for mindreading. This criticism has been dubbed the "logical problem" because it points to a flaw in the logic of the current experimental approach (Hurley and Nudds 2006; Lurz 2011). Given the logical problem, the skeptics maintain that we cannot accept any of the positive evidence for animal mindreading that has been produced thus far.

In this article I defend the position of comparative psychologists against this criticism. ${ }^{2} \mathrm{I}$ begin in section 2 by showing that mindreading skeptics are in agreement with comparative psychologists concerning the definition of mindreading. According to both camps, mindreading is the ability to classify disparate behaviors into abstract equivalence classes according to the cognitive state that they have in common. I then show how comparative psychologists employ what John Stuart Mill identified as the methods of agreement and difference in order to determine whether nonhuman animals have this ability. In the 1990s, most comparative psychologists held that chimpanzees were incapable of mindreading because they did not meet the evidential criteria accepted in the field. However, chimpanzees were subsequently found to meet these criteria, which is the basis for the current consensus. I briefly present this shift in the field in order to illustrate the application of Mill's methods and to distinguish the early opposition to animal mindreading from the current opposition, which is framed in terms of the logical problem.

In section 3 I introduce the logical problem and show how, unlike the early opposition to chimpanzee mindreading, it rejects the methods and evidential criteria currently used in comparative psychology. In section 4 I

2. For other arguments against the position of mindreading skeptics see Andrews (2012), Fletcher and Carruthers (2013), Andrews and Huss (2014), and Buckner (2014). 
present my objections to this new oppositional approach. I argue that the logical problem is a version of the general skeptical problem known as the theoretician's dilemma. As such, it is not a special problem facing those investigating nonhuman animal mindreading, but rather a feature of all theoretical claims. I consider two objections to my argument: first, that it renders mindreading empirically intractable, and second, that it misconstrues the logical problem. I argue that both of these objections fail to restore the logical problem to its current position as a problem that undermines contemporary research on mindreading in nonhuman animals. I conclude that the logical problem is not a problem that comparative psychologists must solve before making claims about mindreading. Thus, mindreading skeptics are not justified in dismissing the experimental methods currently used in comparative psychology or the positive evidence for animal mindreading that these methods have produced. ${ }^{3}$

\section{Identifying Mindreading in Nonhuman Animals}

2.1. What Is Mindreading? Penn and Povinelli (2007) present a minimal definition of mindreading that captures the core of the concept well. In their view, mindreading is the ability to produce and use ms variables, where an ms variable carries information about the cognitive state of another organism. ${ }^{4}$ Since one organism cannot directly observe the cognitive state of another organism, mind readers must produce ms variables on the basis of observable information. That is, they must implement some function that allows them to calculate ms variables on the basis of observable input. How do we determine whether an agent is producing an ms variable? Penn and Povinelli urge, "Let us agree that an ms variable carries information about some other cognitive state if the state of the ms variable covaries with the state of the other cognitive state in a generally reliable manner such that, ceteris paribus, variation in the ms variable can be used by the consuming cognitive system to infer corresponding variations in the cognitive

3. Two notes concerning the scope of this paper are important to emphasize. First, I focus on the mindreading claim that chimpanzees know what objects another agent can or cannot see, given that agent's point of view (what psychologists refer to as "level 1 visual perspective taking"). However, I take my argument against the logical problem to apply to all domains of mindreading research. Second, I do not hold that the logical problem exhausts all arguments against mindreading in nonhuman animals. There are alternative routes that mindreading skeptics could take in formulating their critique, several of which I outline in sec. 4 (see also n. 6). The purpose of this paper is instead to show that one common strategy for arguing against animal mindreading is unsuccessful. 4. Penn and Povinelli (2007) do not state whether the letters "ms" stand for "mental state" or "mindreading state." I prefer the term "mindreading state" because it is not as easily conflated with "cognitive state." 
state" $(2007,733)$. A mind reader, then, uses information that is a function of another organism's cognitive state $(\mathrm{ms}=f(\mathrm{cs}))$.

2.2. How Do Comparative Psychologists Test for Mindreading? The definition of mindreading endorsed by skeptics such as Povinelli and colleagues concurs with the approach that comparative psychologists currently take to determine the mindreading abilities of nonhuman animals. Psychologists test for mindreading abilities by using Mill's methods of agreement and difference (Mill 1872/2006, 388-96). Mill proposed these methods as a way of establishing a regularity or correlation between two phenomena. ${ }^{5}$ Suppose that we would like to determine whether there is a regularity between $x$ and $y$, where $x$ temporally precedes $y$. The method of difference does this by varying $x$ while keeping the context in which $x$ occurs constant. If the presence of $x$ consistently leads to $y$, while its absence consistently does not, then we have evidence that $x$ and $y$ covary. The method of agreement, on the other hand, tests whether there is a relationship between $x$ and $y$ by varying the contexts in which $x$ occurs and seeing whether this has an effect on the occurrence of $y$. If the situations leading to $y$ are such that they have nothing in common but $x$, then we have reason to believe that it was precisely the presence of $x$ that led to the occurrence of $y$.

Comparative psychologists employ these methods in order to determine whether nonhuman animals are sensitive to the cognitive states of other agents. For example, they use them to test whether chimpanzees engage in level 1 visual perspective taking or have the ability to know whether an agent can or cannot see a particular object. Employing the definition of mindreading introduced above, the question here is whether chimpanzees produce and use an ms variable that reliably covaries with an agent's visual state of seeing an object. ${ }^{6}$ To address this question, psychologists apply the method of difference by varying the visual state of some agent (cs) while keeping the background variables $(s)$ constant and examining whether chimpanzees differentially respond to these changes in $\mathrm{cs}^{7}{ }^{7}$ In psychological

5. Mill maintained that the method of agreement could only establish an invariable law between two phenomena, while the method of difference could further establish a causal law between phenomena $(1872 / 2006,394)$. Given our definition of mindreading, however, we need not worry about establishing causal relationships, so I will discuss these methods in terms of demonstrating that two phenomena correlate reliably.

6. Researchers pursue simultaneously the question whether subjects are sensitive to an agent's visual state of not seeing an object.

7. Researchers cannot, of course, directly observe ms since it is an informational state instantiated in an organism. Because of this, they rely on behaviors that are taken to be 
experiments such as these, researchers cannot hold all of the background variables constant. That is, they cannot alter the visual state of an agent without changing some feature of the observable world (such as the orientation of the agent's head, the location of the seen/unseen object, the opacity of a barrier, etc.). Thus, one might worry that these variations in $s$ are leading to differences in $\mathrm{ms}$, rather than the variations in cs. In order to control for this possibility, researchers turn to the method of agreement.

Psychologists apply the method of agreement by varying the background variables associated with cs, while keeping cs constant. In the case of visual perspective taking experiments, this involves employing a variety of techniques for changing the visual state of an agent. An agent might see an object because her eyes are oriented toward it, there is no barrier between her eyes and the object, the barrier between her eyes and the object is transparent, and so on. By showing that a subject is sensitive to cs across a wide range of observable situations, one demonstrates that the subject's ms variable is a function of cs, rather than a particular $s$.

In summary, researchers test for mindreading by applying Mill's methods of difference and agreement. We can present the application of these methods in the form of two mindreading criteria:

1. cs and ms covary;

2. cs is the only variable that is common to all situations leading to ms.

These two criteria pick out mindreading as defined by Penn and Povinelli above. They also pick out mindreading as characterized by Andrew Whiten (1993, 1996, 1998). For Whiten, a mind reader is an individual who classifies disparate behaviors into the same class on the basis of the unobservable cognitive state that they have in common (what is referred to as an "intervening variable"). Under this view, a mind reader with respect to perceptual states should classify all seeing agents together (and non-seeing agents together) even when these agents have no observable features in common. Penn and Povinelli take their definition of mindreading to be consistent with Whiten's account. They write that "being able to recode perceptually disparate behavioural patterns resulting from the same underlying cognitive state as instances of the same abstract equivalence class is a bona fide example of postulating an ms variable in the sense defined hereinabove" $(2007,733)$. Through the application of Mill's methods, com-

reliable indicators of ms. For example, common indicators of the ms variable "I know that you see" include the production of visual gestures in contexts involving cooperative food-bearing agents and the avoidance of food in contexts involving dominant competitors. 
parative psychologists test whether an individual is grouping observable situations in this way. ${ }^{8}$

\subsection{Visual Perspective Taking in Great Apes: The Shift in Consensus.} In the 1990s, many comparative psychologists agreed that chimpanzees were not capable of attributing perceptual states to others. Early experiments by Povinelli and colleagues played an important role in establishing this consensus. Most importantly, Povinelli and Eddy (1996) conducted a series of experiments that showed that chimpanzees failed to meet the two criteria for mindreading specified above. They found that chimpanzee subjects were generally insensitive to the difference between an experimenter who could see them and one who could not, thus failing to meet the first criterion. They also found that, in those cases when subjects appeared to be sensitive to the presence and absence of cs, there was an observable cue that accompanied cs, and the pattern of performance exhibited by subjects suggested that they were responding to this cue rather than to cs. Thus, subjects also failed to meet the second criterion for mindreading. Povinelli and Eddy draw two main conclusions from their studies: "appropriate experimental designs, coupled with sufficiently large sample sizes, can provide a very sensitive analysis of what nonhuman primates know about the mind" (140), and "collectively, our findings provide little evidence that young chimpanzees understand seeing as a mental state" (vi). Throughout the 1990s, most comparative psychologists agreed (Call, Hare, and Tomasello 1998; Tomasello, Hare, and Agnetta 1999).

This consensus shifted at the turn of the century, however. As researchers conducted more mindreading experiments, they found that chimpanzees did in fact meet the two criteria for mindreading with respect to perceptual states (Tomasello, Call, and Hare 2003; Tomasello and Call 2006; Call and Tomasello 2008). For example, they found that chimpanzees treat an agent as "seeing an object $x$ " when that agent directs her gaze at $x$, has

8. It is worth noting that the definition of mindreading advanced by Whiten and by Penn and Povinelli is relatively minimal and thus will not satisfy some mindreading theorists. For example, mindreading in this sense requires only what Josef Perner identifies as secondary representation (the ability to reason about hypothetical situations), not metarepresentation (the ability to represent something as a representation). Thus, those who maintain that the term "mindreading" should be reserved for metarepresentational abilities will not be satisfied with the way that it is employed here (see Perner 1991; Call 2001; Whiten and Suddendorf 2001). Others might find this characterization of mindreading unsatisfying insofar as it presumes a clear divide between reasoning about mental states and behavior (see Gallagher's position in Gallagher and Povinelli 2012). The purpose of this paper, however, is not to argue for a particular definition of mindreading, but rather to show that there is no problem providing evidence for mindreading according to the definition accepted by mindreading skeptics such as Povinelli and colleagues. 
looked at $x$ in the past, or could potentially be looking at $x$ (Hare et al. 2000; Hare, Call, and Tomasello 2001; Hare and Tomasello 2006; Melis, Call, and Tomasello 2006; Kaminski, Call, and Tomasello 2008). A similar result was found for the cognitive state of not seeing: chimpanzees treat an agent as "not seeing an object $x$ " when that agent is facing away from $x$, facing $x$ with closed eyes, facing $x$ with open eyes but with an opaque barrier between the agent and $x$, and so on (Hare et al. 2000, 2001; Hostetter, Cantero, and Hopkins 2001; Hostetter et al. 2007; Leavens, Russell, and Hopkins 2010). Moreover, many of these experiments were specifically designed in order to control for observable cues that typically co-occur with the cognitive states of seeing and not seeing, such as an agent's head orientation or looking behavior. For example, Hare et al. (2001) controlled for the observable cue of an agent orienting toward an object in the recent past, and Kaminski et al. (2008) further controlled for the observable cue of an agent orienting toward a piece of food in its most recent location. Melis et al. (2006) controlled for all looking behavior by requiring that subjects reason about an agent's potential ability to see something, rather than their actual perceptual states. The observable situations in the above experiments were all various (whether or not an object was moved, whether a subject chose first or second in a turn-taking game, whether a tunnel was transparent or opaque), yet subjects treated them as belonging to the same abstract equivalence class based on the cognitive state that they had in common.

In the context of experiments such as those above, the findings of Povinelli and Eddy seemed anomalous, and researchers wondered whether some aspect of their experiment might have unintentionally impeded the performance of their subjects. Thus, Kaminski, Call, and Tomasello (2004) conducted a study with conditions similar to those examined by Povinelli and Eddy, but with improved methods. ${ }^{9}$ This new study found that chimpanzees were more sensitive to states of seeing and not seeing than found in the original study. Other experiments confirmed this finding (Hostetter et al. 2001, 2007; Leavens et al. 2004, 2010; Liebal et al. 2004; Poss et al. 2006; Bräuer, Call, and Tomasello 2007; Hopkins, Taglialatela, and Leavens 2007; Tempelmann, Kaminski, and Liebal 2011).

In this way, the majority of comparative psychologists have gone from holding that apes do not understand perceptual states to thinking that they

9. In Povinelli and Eddy's study, subjects were faced with the task of choosing to beg for food from one of two experimenters. This is not a predicament that chimpanzees usually face, and they had to be trained for hundreds of trials in order to learn that they had to choose between the two experimenters. Thus, Kaminski et al. (2004) designed a study similar to that of Povinelli and Eddy's, except that subjects faced only one experimenter during a trial and their gesturing behavior was compared across trials. Using this approach, the researchers did not need to train the subjects to participate in the experiment. 
do (for reviews, see Tomasello and Call 2006; Call and Tomasello 2008). Whereas in the early stages of mindreading research chimpanzees appeared to fail the two criteria for mindreading, this changed as an increasingly large number of studies showed that they were in fact capable of making the appropriate discriminations in a wide range of contexts. Of course, as Tomasello and Call write, "science is open-ended, and the case is certainly not closed on the issue of whether chimpanzees understand seeing" (2006, 382), but for comparative psychologists the evidence currently favors the hypothesis that they do.

3. The Logical Problem. Povinelli and colleagues' early opposition to nonhuman animal mindreading fit within the traditional experimental methods and evidential criteria employed in comparative psychology. However, the current criticism of mindreading (the logical problem) departs dramatically from this tradition. This criticism maintains that the above experimental approach cannot provide evidence for or against a mindreading hypothesis even in principle (Povinelli and Vonk 2004; Penn and Povinelli 2007).

Proponents of the logical problem maintain that, under the current experimental approach taken by comparative psychologists, there is a complementary behavior-reading (CBR) hypothesis for every mindreading hypothesis. As we have seen, a mindreading hypothesis posits that subjects perform successfully on mindreading experiments because they are capable of predicting an agent's behavior on the basis of information about that agent's cognitive state. For example, a subject might reason, "dominant competitors tend to retrieve food that they can see; this competitor can see the food (cs), so she will retrieve it $(b)$." A CBR hypothesis, in contrast, posits that subjects perform successfully on mindreading experiments because they are capable of predicting an individual's behavior on the basis of observable regularities alone. In such a case, a subject might reason, "dominant competitors tend to retrieve food that is in their direct line of gaze; this food is in the competitor's direct line of gaze $(s)$, so she will retrieve it (b)."

Proponents of the logical problem urge that the current experimental approach used to test for mindreading in nonhuman animals is incapable of distinguishing between mindreading and CBR hypotheses. In order to mindread, an agent requires knowledge of the regularity that some situation $s$ will lead an agent to have the cognitive state cs, which will in turn lead that agent to produce a particular behavior $b(s \rightarrow \mathrm{cs} \rightarrow b)$. But if subjects have knowledge of this regularity, they also have knowledge of the regularity that the observable situation $s$ will lead an agent to produce a particular behavior $b(s \rightarrow b)$. Why posit the additional inferential step involving cs? According to proponents of the logical problem, current mind- 
reading experiments give us no reason to do so. As Penn and Povinelli write, "in order to produce experimental evidence for an $f_{\text {Tом }}$ [theory of mind function] one must first falsify the null hypothesis that the agents in question are simply using their normal, first-person cognitive state variables" $(2007,734)$. However, no mindreading experiment conducted thus far has succeeded in rejecting this null hypothesis. It is not until the CBR hypothesis is rejected that researchers will have provided "compelling evidence for the cognitive (i.e., causal) necessity of an $\mathrm{f}_{\text {ToM }}$ in addition to and distinct from the cognitive work that could have been performed without such a function" (734).

The logical problem maintains that the logic of the current experimental approach fails to reject the hypothesis that subjects solve apparent mindreading tasks on the basis of observable regularities alone. The idea that one must reject the CBR hypothesis before having evidence for mindreading motivates the general strategy employed by skeptics for undermining what comparative psychologists take as evidence for mindreading in nonhuman animals. This strategy runs as follows:

1. Identify those experiments that seem to provide evidence that nonhuman animals mindread.

2. For each experiment identified in step 1 , show that the regularity $s \rightarrow$ $b$ can replace the regularity $s \rightarrow$ cs $\rightarrow b$.

3. On the basis of step 2, maintain that subjects could have solved the experimental task on the basis of having knowledge of $s \rightarrow b$, rather than knowledge of $s \rightarrow$ cs $\rightarrow b$.

4. On the basis of step 3, conclude that one cannot reject the null hypothesis that subjects solved the experimental task using their knowledge of $s \rightarrow b$.

5. Conclude that there is no evidence that nonhuman animals mindread.

Before presenting my critique of this strategy, there are three things that are important to note. First, in employing this strategy, skeptics are not challenging the claim that nonhuman animals meet the first mindreading criterion (that cs and ms covary). Penn and Povinelli (2009) agree that nonhuman animals such as apes often behave exactly as if they have knowledge of certain cognitive states. In fact, they go so far as to state that with regard to the early competition studies of Hare and colleagues (Hare et al. 2000, 2001), "there has never been any dispute about the fact that chimpanzees act as if they understand that others can see things" (Penn and Povinelli 2009, 17). However, when psychologists say that there is evidence that chimpanzees produce and employ an ms variable, they mean nothing more than that there is behavioral evidence that this is the case. Thus, both parties 
agree that chimpanzees behave as if they produce and use an ms variable that covaries with the perceptual states of other agents.

Second, the skeptics are not challenging the claim that nonhuman animals meet the second mindreading criterion (that cs is the only variable common to the situations leading to $\mathrm{ms}$ ). When showing how one can appeal to some regularity $s \rightarrow b$, mindreading skeptics rarely try to show that the $s$ in each experiment is the same. The antecedent variables can be various, and the regularities can be abstract and complex. Indeed, Povinelli and colleagues argue that given what is known about nonhuman primate behavior in experimental and naturalistic settings, we have reason to think that these regularities will be abstract and complex (Povinelli and Vonk 2004; Penn and Povinelli 2013).

Third, in employing the above strategy, mindreading skeptics do not attempt to show that it is ontogenetically or phylogenetically more plausible that subjects are employing $s \rightarrow b$ over $s \rightarrow$ cs $\rightarrow b$. Instead, they observe that if an individual has knowledge of $s \rightarrow \mathrm{cs} \rightarrow b$, then that individual also has knowledge of $s \rightarrow b$. Thus, the claim that a subject has somehow acquired $s \rightarrow b$ is no less empirically plausible than the claim that this subject has somehow acquired $s \rightarrow \mathrm{cs} \rightarrow b$. As Povinelli and Vonk write, "the problem we face is not primarily an empirical one. Instead, the most pressing problem is to come to grips with the fact that the experimental results from the kinds of techniques that are currently in vogue cannot add a single bit of evidence in unique support of the conclusion that chimpanzees reason about mental states - any mental states" (2004, 11; emphasis in the original). ${ }^{10}$

Some mindreading skeptics might deny the above characterization of the logical problem by denying one or more of the three points above. That is, they could argue that (1) the logical problem is in fact the claim that nonhuman animals fail to meet one or both of the mindreading criteria or (2) the CBR hypothesis is actually a competing empirical hypothesis, rather than a null hypothesis. I discuss these options in the following section, arguing that although they allow one to avoid the criticism that the logical problem is a general skeptical problem, they transform the problem into one

10. It is worth noting that Povinelli and colleagues do provide an evolutionary account aimed at motivating the claim that nonhuman animals are incapable of mindreading. Their "reinterpretation hypothesis" posits that nonhuman primates have adapted to the observable regularities that result from cognitive agents interacting with the world, but that only humans evolved the additional ability to reinterpret these observable regularities in terms of the unobservable cognitive states underlying them (Povinelli and Vonk 2003; Vonk and Povinelli 2006). However, this evolutionary account is not aimed at making the CBR hypothesis more plausible than the mindreading hypothesis; instead, it is aimed at motivating the possibility that nonhuman animals behave like mind readers while lacking mindreading abilities. 
that is ineffective at undermining the experimental approach currently used in comparative psychology.

4. Why the Logical Problem Is Not a Special Problem. In this section I argue that the logical problem is a version of what Hempel identified as the theoretician's dilemma. As such, it is not a special problem facing comparative psychologists that can be solved through the design of better experiments. I then consider two objections to this argument: first, that it renders mindreading empirically intractable, and second, that it mischaracterizes the CBR hypothesis. Both of these objections, I argue, fail to restore the logical problem to its original status as a problem that undermines current mindreading research.

The fact that the logical problem (as characterized in the previous section) is not solvable can be seen when one takes seriously the claim that mindreading is not an act of telepathy. Given that mind readers are not telepathic, any individual capable of mindreading must do so on the basis of inferring cs from some observable state of affairs $(s)$. Moreover, an accurate mind reader will infer cs on the basis of those observable situations that actually lead to cs. In the case of visual perspective taking, for example, an accurate mind reader will infer that another agent can see $x$ in those situations in which the agent can actually see $x$ (such as situations in which the agent's eyes are open, there is no opaque barrier between the agent and $x$, etc.). Of course, one will not have knowledge of all of the observable situations that lead to cs. Instead, a mind reader will only be able to infer cs on the basis of the knowledge that he has uniquely acquired over evolutionary and developmental time. That is, an individual can only infer cs on the basis of some subset of $s$, a subset that reflects the organism's innate and acquired knowledge of those situations that lead to cs. Thus, within a given experiment, the ms of an organism is not a function of cs or $s$, but rather some subset of $s$ that the organism knows reliably leads to cs. If this were not the case, then mind readers would either have to infer cs without any grounds for doing so or have to infer cs directly (that is, telepathically).

Given the above, one can always show that a subject is solving a particular mindreading task on the basis of observable regularities alone. In order for subjects to perform successfully on a mindreading task, they must know that $s \rightarrow$ cs because without such knowledge they would have no reason to infer cs on the basis of the experimental situation. However, they must also know that cs $\rightarrow b$. Without this knowledge, subjects would be unable to make the behavioral predictions necessary to demonstrate their understanding of another agent's cognitive state. Both of these elements are required in order to get any mindreading experiment off the ground. However, if subjects know $s \rightarrow$ cs and cs $\rightarrow b$, then they know that $s \rightarrow$ cs $\rightarrow b$. And if they know this, then they also know that $s \rightarrow b$. Thus, the min- 
imal knowledge required to solve a mindreading task is all that is needed in order to make the case that an individual solved that task using CBR instead. If one has positive results for mindreading, one has also failed to reject the CBR hypothesis.

The idea that one can replace a regularity involving theoretical terms with a regularity devoid of such terms is what Hempel identified as the "theoretician's dilemma." As Hempel writes, "if the terms of and the general principles of a scientific theory serve their purpose, i.e., if they establish definite connections among observable phenomena, then they can be dispensed with since any chain of laws and interpretative statements establishing such a connection should then be replaceable by a law which directly links observational antecedents to observational consequents" $(1958,49)$. In other words, if one uses theoretical terms to successfully establish a regularity among observable phenomena, then one can remove those theoretical terms from one's account and describe the regularity in terms of observable phenomena alone. Similarly, we can identify a mind reader's dilemma:

If an individual is capable of mindreading, i.e., if she establishes definite connections among observable phenomena, then her mindreading abilities can be dispensed with since any evolved or learned rule establishing such a connection should then be replaceable by a rule that directly links observational antecedents to observational consequents.

The logical problem simply is the mind reader's dilemma. Given this, any experiment aimed at testing mindreading will fail to reject the hypothesis that subjects are reasoning in terms of observable regularities alone.

To be clear, the mind reader's dilemma is a modified version of the theoretician's dilemma — one that takes the mind reader to be the theoretician. It is important to recognize the difference between these two dilemmas. The theoretician's dilemma is a claim about the theoretical terms posited by researchers. If the logical problem were an unmodified version of the theoretician's dilemma, then this would suggest that mindreading skeptics are skeptical of the use of theoretical concepts to explain nonhuman animal behavior. Such skepticism would be akin to behaviorism, requiring that researchers reformulate hypotheses of animal behavior in terms of observable variables alone. Crucially, this is not the mindreading skeptics' position. They are not skeptical of the theoretical concepts employed by comparative psychologists, but rather those employed by nonhuman animal subjects.

The mind reader's dilemma captures this skepticism, holding that it is always possible to replace the theoretical concepts employed by mind readers with observable variables. Such a move transforms a mindreading hypothesis into a behavior-reading one. However, the behavior-reading hypothesis itself still contains theoretical terms. It posits, for example, that 
nonhuman animal subjects have certain cognitive abilities, such as the ability to form memories and abstract behavioral rules. It simply denies that nonhuman animals reason about the unobservable mental states of other agents.

In the literature, mindreading skeptics present the logical problem as a methodological problem that is solvable. They argue that it is not the investigation of mindreading that is problematic, but rather the experimental approach that comparative psychologists have used to investigate it thus far. This experimental approach, they argue, needs to be replaced with a new methodology, one capable of rejecting the CBR hypothesis. Several such new methodologies have been proposed (see Heyes 1998; Povinelli and Vonk 2004; Penn and Povinelli 2007; Lurz 2011; Lurz and Krachun 2011). However, none of these have provided a compelling solution to the logical problem (Andrews 2005; Lurz 2009, 2011; Buckner 2014). The fact that such a solution has not been forthcoming makes sense in light of the mind reader's dilemma. Given this dilemma, we should not expect to be able to reject the CBR hypothesis on the basis of the results of an individual experiment, no matter how well it is designed.

Does the fact that the logical problem is a general skeptical problem render mindreading empirically intractable? Crucially, it does not. Instead, it requires that one take a different approach to the problem. For any experiment considered in isolation, ms will covary with some observable state of affairs. Thus, for any individual experiment, there is no way to determine whether subjects are responding to cs or the corresponding observable state. Comparative psychologists, however, can get around the mind reader's dilemma by applying Mill's method of agreement to a collection of experiments. Doing so provides evidence for mindreading insofar as it establishes that subjects are responding to a diverse set of observable variables (eyes closed, opaque barrier present, head turned) as belonging to the same abstract equivalence class (situations that lead to a state of not seeing). It is the sensitivity to the existence of this class of behaviors that shows that subjects are reasoning in a way that goes beyond reasoning about observable variables alone.

The above is an instance of causal reasoning that is used widely in the sciences. Mill's methods are generally used to identify the likely cause of an event from a set of possibilities. Discovering that there is one variable common to all situations leading to an effect gives us reason to believe that it is this variable that is causally related to that effect. In the case of mindreading, researchers need not go so far as to establish that cs causes ms; it is enough to show that cs and ms covary in a reliable manner (see sec. 2.1). Positing that chimpanzees are reasoning about unobservable mental states is justified, then, insofar as this variable leads to the same effect across a wide range of observable conditions. Experiments on visual perspective taking in chimpanzees have demonstrated precisely this. 
One could attempt to apply something like the logical problem to a collection of experiments. That is, one could argue that in order to have evidence for mindreading, one must show that it is plausible that subjects are classifying situations together on the basis of the underlying cognitive state that they have in common and not on the basis of some observable cue that they have in common. Lurz (2011), for example, argues that one can account for many of the results of visual perspective taking experiments by positing that subjects reason in terms of a "direct line of gaze" rather than the mental state of seeing. I agree that if mindreading skeptics could show that there were some observable cue common to all mindreading experiments aimed at testing a subject's knowledge of a particular cognitive state, then this would indeed undermine the claim that these experiments provide evidence for mindreading. However, comparative psychologists are well aware of this and control for it by applying the method of agreement (as discussed in sec. 2.3). It is precisely because there is no such cue common to all situations involving cs that researchers hold that there is evidence for mindreading. The logical problem in this guise does not undermine the current experimental approach. Thus, if mindreading skeptics take this to be the logical problem, they cannot also maintain, as they currently do, that the current experimental approach cannot provide evidence for mindreading even in principle.

A second objection to the above argument is that it mischaracterizes the nature of the CBR hypothesis. Mindreading skeptics might concede that, understood as a null hypothesis, the CBR hypothesis cannot be rejected because one can always reinterpret mindreading as the ability to reason about observable regularities. However, the CBR hypothesis is not a null hypothesis, but rather a positive empirical claim with its own evidential support and epistemic virtues. The logical problem is really the problem that the mindreading and CBR hypotheses are equivalent in their theoretical plausibility and evidential support.

There are two responses to this objection. First, the CBR hypothesis does not have the features of an empirical hypothesis and is generally not advanced by skeptics as such. As discussed in section 3, skeptics do little to motivate the CBR hypothesis empirically. More importantly, however, the CBR hypothesis lacks the content necessary to make new predictions. As Fletcher and Carruthers point out, "the behavior-rule account is only capable of 'predicting' new findings after they are discovered, postulating a novel behavior-rule for the purpose" $(2013,8)$. Mindreading skeptics have yet to provide a positive account concerning what behavior-reading skills we should expect particular nonhuman animals to possess independently of their performance on mindreading tasks. Instead, the behavior-reading skills proposed are all and only those that can account for the current results of mindreading research. 
Second, if the CBR hypothesis were an empirical hypothesis, then it would not give rise to a logical problem. If mindreading skeptics were to begin motivating the CBR hypothesis on grounds other than those provided by the results of mindreading experiments, we could begin discerning the differences between these two hypotheses. We could assess the relative virtues of the accounts based on, for example, their ability to account for the empirical data, coherence with other theories, neurobiological plausibility, and so on. If this were the case, though, mindreading skeptics could no longer follow the general strategy (outlined in sec. 3) that they currently use to undermine the positive results of mindreading experiments. Simply showing that one can account for the successful performance of subjects on a given mindreading task by positing that they employ the relevant $s \rightarrow b$ rules would not undermine these results or their interpretation. If the CBR hypothesis were a null hypothesis, then such a move would indeed show that these experiments fail to provide evidence for mindreading. As an empirical hypothesis, however, this strategy does no more than illustrate the underdetermination of theories by evidence. What is needed is an account of what exactly the CBR hypothesis claims about the biological, psychological, and behavioral capacities of apes and why these claims are theoretically and empirically more plausible than positing that apes mindread. Without such an account, the CBR hypothesis is not one that we can evaluate as a serious alternative to mindreading.

Hempel rejects the theoretician's dilemma, arguing that just because one can get rid of the theoretical terms in an explanation does not mean that one should. Instead, he maintains that researchers must take others things into account, such as whether the presence or absence of the theoretical terms leads to progress, given one's epistemic goals. I suggest that we reject the mind reader's dilemma on similar grounds. The fact that we can account for chimpanzee social behavior without positing mindreading abilities does not mean that we should. Instead, we must evaluate both the mindreading and CBR hypotheses in light of their success relative to the epistemic goals valued by researchers. Currently, such an evaluation favors the mindreading hypothesis. The logical problem poses no threat to this claim.

5. Conclusion. Comparative psychologists test for mindreading in nonhuman animals by determining whether they detect the presence and absence of particular cognitive states in a wide variety of circumstances. They eliminate potential confounding variables by ensuring that there is no one observable state to which subjects might be responding. In the 1990s, Povinelli and colleagues agreed with this methodological approach. Indeed, they advocated it. However, over the past decade, they and other skeptics have argued that animals might be solving mindreading tasks on the basis of knowledge of observable regularities alone; thus, most of the mind- 
reading research conducted to date must be dismissed as methodologically flawed. I have argued that this new oppositional strategy is mistaken. In the same way that one can reinterpret a successful theory in terms of regularities among observables, one can always reinterpret a successful mind reader as a complementary behavior reader.

Penn and Povinelli (2013) object that they are sometimes accused of "derived behaviorism" and that this accusation depends on a straw man of their account, one that casts them as holding that nonhuman animals have no representational abilities or are purely Pavlovian learners. I agree that this is not their position. However, the arguments that they advance may have more in common with behaviorism than they might like to admit. Skinner criticized the practice in psychology of positing unobservable variables as intervening between two observable entities in a causal chain. He argued that if there is a regular relationship between observable and unobservable variables, then there is also a regular relationship between the observable variables alone; thus, the unobservable variable is causally superfluous. Indeed, it was this claim of Skinner's that led Hempel to articulate the theoretician's dilemma (see Hempel 1958, 49). Thus, the logical problem constitutes a sort of second-order behaviorism - behaviorism not with respect to our scientific understanding of agents, but with respect to nonhuman animals' understanding of other agents. Neither form of behaviorism, I believe, will help us develop an empirically informed account of animal psychology.

\section{REFERENCES}

Andrews, Kristin. 2005. "Chimpanzee Theory of Mind: Looking in All the Wrong Places?" Mind and Language 20 (5): 521-36.

2012. Review of Mindreading Animals: The Debate over What Animals Know about Other Minds, by Robert W. Lurz. Notre Dame Philosophical Reviews.

$\rightarrow$ Andrews, Kristin, and Brian Huss. 2014. "Anthropomorphism, Anthropectomy, and the Null Hypothesis." Biology and Philosophy 29:711-29.

$\rightarrow$ Bräuer, Juliane, Josep Call, and Michael Tomasello. 2007. "Chimpanzees Really Know What Others Can See in a Competitive Situation." Animal Cognition 10:439-48.

$\rightarrow$ Buckner, Cameron. 2014. "The Semantic Problem(s) with Research on Animal Mind-Reading." Mind and Language 29 (5): 566-89.

$\rightarrow$ Call, Josep. 2001. “Chimpanzee Social Cognition.” TRENDS in Cognitive Science 5 (9): 388-93.

$\rightarrow$ Call, Josep, Brian Hare, and Michael Tomasello. 1998. "Chimpanzee Gaze Following in an ObjectChoice Task." Animal Cognition 1 (2): 89-99.

$\rightarrow$ Call, Josep, and Michael Tomasello. 2008. "Does the Chimpanzee Have a Theory of Mind? 30 Years Later.” TRENDS in Cognitive Sciences 12 (5): 187-92.

Callender, Craig, and Jonathan Cohen. 2006. "There Is No Special Problem about Scientific Representation." Theoria 55:7-25.

Fletcher, Logan, and Peter Carruthers. 2013. "Behavior-Reading versus Mentalizing in Animals." In Agency and Joint Attention, ed. Janet Metcalfe and Herbert S. Terrace, 82-99. Oxford: Oxford University Press. 
$\rightarrow$ Gallagher, Shaun, and Daniel J. Povinelli. 2012. "Enactive and Behavioral Abstraction Accounts of Social Understanding in Chimpanzees, Infants, and Adults." Review of Philosophy and Psychology 3:145-69.

$\rightarrow$ Hare, Brian, Josep Call, Bryan Agnetta, and Michael Tomasello. 2000. "Chimpanzees Know What Conspecifics Do and Do Not See.” Animal Behaviour 59:771-85.

$\rightarrow$ Hare, Brian, Josep Call, and Michael Tomasello. 2001. "Do Chimpanzees Know What Conspecifics Know?" Animal Behaviour 61:139-51.

Hare, Brian, and Michael Tomasello. 2006. "Chimpanzees Deceive a Human by Hiding." Cognition 101 (3): 495-514.

Hempel, Carl G. 1958. "The Theoretician's Dilemma: A Study in the Logic of Theory Construction." In Minnesota Studies in the Philosophy of Science, ed. Herbert Feigl, Michael Scriven, and Grover Maxwell, 37-98. Minneapolis: University of Minnesota Press.

Heyes, Cecilia. 1998. "Theory of Mind in Nonhuman Primates." Behavioral and Brain Sciences 21:101-48.

$\rightarrow$ Hopkins, William, Jared P. Taglialatela, and David A. Leavens. 2007. "Chimpanzees Differentially Produce Novel Vocalizations to Capture the Attention of a Human.” Animal Behaviour 73 (2): 281-86.

$\rightarrow$ Hostetter, Autumn B., Monica Cantero, and William D. Hopkins. 2001. "Differential Use of Vocal and Gestural Communication by Chimpanzees (Pan troglodytes) in Response to the Attentional Status of a Human (Homo sapiens)." Journal of Comparative Psychology 115 (4): 337-43.

Hostetter, Autumn B., Jamie L. Russell, Hani Freeman, and William D. Hopkins. 2007. "Now You See Me, Now You Don't: Evidence That Chimpanzees Understand the Role of the Eyes in Attention." Animal Cognition 10 (1): 55-62.

Hurley, Susan, and Matthew Nudds. 2006. "The Question of Animal Rationality: Theory and Evidence.” In Rational Animals?, ed. Susan Hurley and Matthew Nudds, 1-83. Oxford: Oxford University Press.

$\rightarrow$ Kaminski, Juliane, Josep Call, and Michael Tomasello. 2004. "Body Orientation and Face Orientation: Two Factors Controlling Apes’ Begging Behavior from Humans.” Animal Cognition 7:216-23.

$\rightarrow$ 2008. "Chimpanzees Know What Others Know, but Not What They Believe." Cognition 109:224-34.

Leavens, David A., Autumn B. Hostetter, Michael J. Wesley, and William D. Hopkins. 2004. "Tactical Use of Unimodal and Bimodal Communication by Chimpanzees, Pan troglodytes." Animal Behaviour 67:467-76.

Leavens, David A., Jamie L. Russell, and William D. Hopkins. 2010. "Multimodal Communication by Captive Chimpanzees (Pan troglodytes)." Animal Cognition 13 (1): 33-40.

Liebal, Katja, Simone Pika, Josep Call, and Michael Tomasello. 2004. "To Move or Not to Move: How Apes Adjust to the Attentional State of Others." Interaction Studies 5 (2): 199-219.

$\rightarrow$ Lurz, Robert. 2009. "If Chimpanzees Are Mindreaders, Could Behavioral Science Tell? Toward a Solution to the Logical Problem." Philosophical Psychology 22 (3): 305-28.

_. 2011. Mindreading Animals: The Debate over What Animals Know about Other Minds. Cambridge, MA: MIT Press.

Lurz, Robert W., and Carla Krachun. 2011. "How Could We Know Whether Nonhuman Primates Understand Others' Internal Goals and Intentions? Solving Povinelli's Problem.” Review of Philosophy and Psychology 2 (3): 449-81.

$\rightarrow$ Melis, Alicia P., Josep Call, and Michael Tomasello. 2006. "Chimpanzees (Pan troglodytes) Conceal Visual and Auditory Information from Others.” Journal of Comparative Psychology 120 (2): $154-62$.

Mill, John Stuart. 1872/2006. The Collected Works of John Stuart Mill. Vol. 7, A System of Logic, Ratiocinative and Inductive, ed. John M. Robson. Repr. Indianapolis: Liberty Fund.

Nichols, Shaun, and Stephen P. Stich. 2003. Mindreading: An Integrated Account of Pretence, SelfAwareness, and Understanding Other Minds. Oxford: Oxford University Press.

Penn, Derek C. 2011. "How Folk Psychology Ruined Comparative Psychology and What Scrub Jays Can Do about It." In Animal Thinking: Contemporary Issues in Comparative Cognition, ed. Randolf Menzel and Julia Fischer, 253-65. Cambridge, MA: MIT Press. 
Penn, Derek C., Keith J. Holyoak, and Daniel J. Povinelli. 2008. "Darwin's Mistake: Explaining the Discontinuity between Human and Nonhuman Minds." Behavioral and Brain Sciences 31 (2): 109-78.

$\rightarrow$ Penn, Derek C., and Daniel J. Povinelli. 2007. "On the Lack of Evidence That Chimpanzees Possess Anything Remotely Resembling a 'Theory of Mind.'” Philosophical Transactions of the Royal Society B 362:731-44.

- 2009. "On Becoming Approximately Rational: The Relational Reinterpretation Hypothesis." In Rational Animals, Irrational Humans, ed. Shigeru Watanabe, Aaron P. Blaisdell, Ludwig Huber, and Allan Young, 23-44. Tokyo: Keio University Press.

. 2013. "The Comparative Delusion: Beyond Behavioristic and Mentalistic Explanations for Nonhuman Social Cognition." In Agency and Joint Attention, ed. Janet Metcalfe and Herbert S. Terrace, 62-81. Oxford: Oxford University Press.

Perner, Josef. 1991. Understanding the Representational Mind. Cambridge, MA: MIT Press.

$\rightarrow$ Poss, Sarah R., Chris Kuhar, Tara S. Stoinski, and William D. Hopkins. 2006. "Differential Use of Attentional and Visual Communicative Signaling by Orangutans (Pongo pygmaeus) and Gorillas (Gorilla gorilla) in Response to the Attentional Status of a Human." American Journal of Primatology 68 (10): 978-92.

$\rightarrow$ Povinelli, Daniel J., and Timothy J. Eddy. 1996. "What Young Chimpanzees Know about Seeing." Monographs of the Society for Research in Child Development 61 (3): 1-152.

$\rightarrow$ Povinelli, Daniel J., and Jennifer Vonk. 2003. “Chimpanzee Minds: Suspiciously Human?” TRENDS in Cognitive Sciences 7 (4): 157-60.

$\rightarrow \quad$. 2004. "We Don't Need a Microscope to Explore the Chimpanzee's Mind." Mind and Language 19 (1): 1-28.

$\rightarrow$ Premack, David, and Guy Woodruff. 1978. "Does the Chimpanzee Have a Theory of Mind?" Behavioral and Brain Sciences 1 (4): 515-26.

Tempelmann, Sebastian, Juliane Kaminski, and Katja Liebal. 2011. "Focus on the Essential: All Great Apes Know When Others Are Being Attentive." Animal Cognition 14 (3): 433-39.

Tomasello, Michael, and Josep Call. 2006. "Do Chimpanzees Know What Others See-or Only What They Are Looking At?" In Rational Animals?, ed. Susan Hurley and Matthew Nudds, 371-84. Oxford: Oxford University Press.

$\rightarrow$ Tomasello, Michael, Josep Call, and Brian Hare. 2003. "Chimpanzees Understand Psychological States - the Question Is Which Ones and to What Extent." TRENDS in Cognitive Sciences 7 (4): 153-56.

Tomasello, Michael, Brian Hare, and Bryan Agnetta. 1999. "Chimpanzees, Pan troglodytes, Follow Gaze Direction Geometrically.” Animal Behaviour 58:769-77.

Vonk, Jennifer, and Daniel J. Povinelli. 2006. "Similarity and Difference in the Conceptual Systems of Primates: The Unobservability Hypothesis.” In Comparative Cognition: Experimental Exploration of Animal Intelligence, ed. Edward A. Wasserman and Thomas R. Zentall, 363 87. New York: Oxford University Press.

Whiten, Andrew. 1993. "Evolving a Theory of Mind: The Nature of Non-verbal Mentalism in Other Primates." In Understanding Other Minds: Perspectives from Autism, ed. Simon BaronCohen, Helen Tager-Flusberg, and Donald J. Cohen, 367-96. Oxford: Oxford University Press.

- 1996. "When Does Smart Behavior-Reading Become Mind-Reading?" In Theories of Theories of Mind, ed. Peter Carruthers and Peter K. Smith, 277-92. Cambridge: Cambridge University Press.

$\rightarrow$. 1998. "Triangulation, Intervening Variables, and Experience Projection." Behavioral and Brain Sciences 21:132-33.

$\rightarrow$ Whiten, Andrew, and Thomas Suddendorf. 2001. "Meta-representation and Secondary Representation." TRENDS in Cognitive Science 5 (9): 378. 